\title{
Enalaprilato na Prevenção da Hipertrofia Ventricular Esquerda Induzida pelo Isoproterenol
}

\author{
Eduardo A. S. Costa, Bráulio Luna Fon, Rui Póvoa, Celso Ferreira Foํ, N eif Murad, \\ Marcelo Ferreira, Celso Ferreira \\ São Paulo, SP
}

\begin{abstract}
Objetivo - Avaliar se o enalaprilato, droga inibidora da enzima de conversão da angiotensina I, previne a hipertrofia ventricular esquerda (HVE) induzida pelo isoproterenol.

Métodos - Foram divididos em 4 grupos, 72 ratos Wistar-EPM: CON controle; ENA, tratados com enalaprilato ( $1 \mathrm{mg} / \mathrm{kg}$ via subcutânea (sc) por 8 dias); ISO, tratados com isoproterenol $(0,3 \mathrm{mg} / \mathrm{kg}$ via sc/8 dias) $e$ ENA+ISO, tratados simultaneamente com ambas as drogas. Em 10 animais de cada grupo foram determinadas a freqüência cardíaca $(F C)$ e a pressão arterial $(P A)$ e verificado o peso de ventrículo esquerdo (VE). Em 8 animais de cada grupo, fragmento do VE foi corado com hematoxilina-eosina e picro-sírius e preparado para estudo morfométrico e ultra-estrutural, respectivamente, com microscópio de luz e eletrônico.
\end{abstract}

Resultados - Nos grupos estudados (CON, ENA, ISO e ISO+ENA) não ocorreram variações na PA. Os grupos ISO e ISO+ENA exibiram aumentos significantes na FC. $O$ grupo ISO apresentou aumento significativo do peso do $V E(P U=0,821 \mathrm{~g}$ e $P S=0,204 \mathrm{~g})$, quando comparado ao grupo CON. O grupo ENA não exibiu modificação de peso do VE quando comparado ao grupo CON (PU $=0,590 \mathrm{~g} e$ $P S=0,139 \mathrm{~g})$. No grupo ENA $+I S O(P U=0,737 \mathrm{~g}$ e $P S=$ $0,177 \mathrm{~g}$ ) constatou-se diferença de peso ao ser comparado aos grupos ISO e CON. A análise morfométrica e ultra-estrutural mostraram que o ISO induziu hipertrofia dos cardiomiócitos e aumento do tecido conjuntivo com depósito de fibras colágenas do tipo I. O enalaprilato associado com isoproterenol atenuou importantemente aquela manifestação.

Conclusão - O enalaprilato inibiu a ação do isoproterenol sobre os cardiomiócitos, evitando parcialmente, na dose utilizada, a HVE e diminuindo também a quantidade de fibras colágenas.

Palavras-chave: hipertrofia ventricular, isoproterenol, enalaprilato

\section{Enalaprilat Prevents the Left Ventricular Hypertrophy Induced by Isoproterenol}

Purpose - To evaluate whether the enalaprilat, angiotensin I enzyme conversion inhibitor, could prevent the left ventricular hypertrophy $(L V H)$ induced by isoproterenol.

Methods - Seventy two adult Wistar-EPM rats were divided into four groups: CON, control; ENA, treated with enalaprilat ( $1 \mathrm{mg} / \mathrm{kg}$ via subcutaneous (sc) for 8 days); ISO, treated with isoproterenol (0.3mg via sc for 8 days) $e$ $E N A+I S O$, treated with both drugs simultaneously. Each group had the arterial blood pressure, cardiac rate and the left ventricle $(L V)$ weight determined in 10 animals. In 8 animals from each group a small sample was taken from the LV and stained with hematoxyline-eosine and picrosirius for morphometric and ultra-structural studies with optic and transmission electronic microscopy.

Results - The ISO group showed that the LV weight increased $47 \%$ in comparison with control. On the other hand the ENA+ISO group showed only $22.1 \%$ increase ( $p \leq 0.05)$. The morphometric and ultra-structural analyses revealed that isoproterenol induced cardiomyocite hypertrophy and augmented the content of the type I collagen in the cardiac interstitium.

Conclusion - Enalaprilat inhibited the isoproterenol action on the cardiomyocite, avoiding partially the LVH and decreasing the content of collagen fibers.

Key-words: ventricular hypertrophy, isoproterenol, enalaprilat

Arq Bras Cardiol, volume 69 (n' 1), 35-39, 1997

Escola Paulista de Medicina-UNIFESP - São Paulo

Correspondência: Eduardo A. S. Costa - Rua Leandro Dupret, 317 - 04025-011 São Paulo, SP

Recebido para publicação em 2/10/96

Aceito 21/5/97
A hipertrofia ventricular esquerda (HVE) é um dos mais importantes fatores de risco nas doenças cardiovasculares. Paralelamente aos fatores de risco tradicionais, como tabagismo, dislipidemia e hipertensão arterial 
sistêmica (HAS), cada vez mais se enfatizam a prevenção e o tratamento da $\mathrm{HVE}^{1}$.

Do ponto de vista morfológico, a HVEé um processo dependente do aumento de volume dos cardiomiócitos pela síntese de novas unidades de sarcômeros e da proliferação de fibras colágenas e elásticas, constituintes fundamentais da arquitetura miocárdica ${ }^{2}$. $\mathrm{O}$ aumento progressivo da concentração de colágeno parece exercer uma série de efeitos adversos no comportamento mecânico do coração, prejudicando o relaxamento diastólico, interferindo na transmissão de forças para as fibras musculares adjacentes e, finalmente, o desenvolvimento de tensão durante a sístole. Além disso, a acentuação do processo fibrótico poderá também induzir atrofia dos cardiomiócitos, criando um cenário de isquemia e isolamento elétrico dessas células, facilitando a instalação de substrato anatômico para o desenvolvimento de arritmias e outras manifestações cardíacas ${ }^{3,4}$.

Vários modelos experimentais têm sido utilizados no estudo HVE. Os mais usados induzem elevação da pressão arterial (PA), provocando uma sobrecarga ventricular ${ }^{5-7}$. Alternativamente, pode-se produzir a HVE através de mecanismo não-pressórico ou humoral que não eleva a PA como, por exemplo, o isoproterenol ${ }^{8,9}$. Esta droga ativa os receptores beta-adrenérgicos, diminuindo a resistência vascular periférica e a PA diastólica, estimulando mecanismos bioquímicos e moleculares celulares moduladores da HVE. Há evidência que este processo complexo conta com a participação de vários mediadores, destacando-se, além da ativação dos receptores-beta e da proteína $\mathrm{G}$, o incremento do $\mathrm{Ca}^{++}$intracelular, do AMP cíclico, da fosfolipase e quinase protéica $\mathrm{C}$, culminando com a ativação de vários fatores de transcrição nuclear, como os protooncogenes c-myc, c-jus e c-fos ${ }^{10-12}$.

O objetivo principal deste trabalho foi avaliar se o enalaprilato, droga inibidora da enzima de conversão da angiotensina (ECA), pode prevenir a HVE induzida pelo isoproterenol, analisando, também, o comportamento dos cardiomiócitos e a distribuição das fibras colágenas no miocárdio.

\section{Métodos}

Foram utilizados 72 ratos (rattus novergicus albinus) da linhagem Wistar-EPM, machos, pesando entre 250 a $300 \mathrm{~g}$, distribuídos ao acaso em quatro grupos. Os animais do controle (CON) foram submetidos a doses subcutâneas (sc) diárias de $\mathrm{lmL} / \mathrm{kg}$ de soro fisiológico (SF) às 8 h e óleo de oliva $1 \mathrm{~mL} / \mathrm{kg}$ às $18 \mathrm{~h}$. O grupo tratado com enalaprilato (ENA) foi submetido a dose de $\mathrm{lmL} / \mathrm{kg}$ de enalaprilato, em solução $\mathrm{de} \mathrm{lmg} / \mathrm{mL}$ às $8 \mathrm{~h}$ e óleo de oliva de forma similar ao controle às $18 \mathrm{~h}$. $\mathrm{O}$ grupo tratado com isoproterenol (ISO) recebeu dose de $\mathrm{SF}$ igual ao grupo $\mathrm{CON}$ às $8 \mathrm{~h}$ e isoproterenol em óleo de oliva, $1 \mathrm{~mL} / \mathrm{kg} \mathrm{sc}$, em solução de $0,3 \mathrm{mg} / \mathrm{mL}$ às $18 \mathrm{~h}$. $\mathrm{O}$ grupo tratado com enalaprilato e isoproterenol (ENA +ISO) recebeu dose de enalaprilato, igual a do grupo ENA às $8 \mathrm{~h}$ e dose de isoproterenol similar ao grupo ISO às $18 \mathrm{~h}$. O período experimental para todos os grupos foi de oito dias.
Em cada grupo, 10 animais foram separados para determinação do peso do ventrículo esquerdo (VE), freqüência cardíaca (FC) e PA. Os corações foram retirados com imediata separação dos átrios e do ventrículo direito, mantendo o septo interventricular como parte do VE, determinando-se imediatamente o peso úmido em balança analítica. A seguir, esses corações foram colocados em estufa por $24 \mathrm{~h} \mathrm{a} 100^{\circ} \mathrm{C}$, avaliando-se o peso seco.

Para a determinação da PA sistêmica e da FC, no dia anterior ao término do experimento, em 10 animais de cada grupo foram instalados, após anestesia com éter etílico, cateter de polietileno (PE-50) da Clay Adams, na artéria ilíaca interna direita por 24h. Após este período, registrados em polígrafo Gould 2200S a PA e a FC.

Em oito animais de cada grupo, ao final do estudo, foram retirados os corações ainda na vigência de contrações e obtidos fragmentos para microscopia de luz e eletrônica de transmissão ${ }^{13,14}$. Os cortes foram corados em hematoxilinaeosina (HE) para análise morfológica e histometria ${ }^{14}$. Também foi utilizado a coloração com hematoxilina-picro-sírius para observação do tipo de colágeno ${ }^{15}$.

As variáveis contínuas estão apresentadas como média e desvio-padrão. Na avaliação dos resultados foi aplicado o teste de análise de variância por posto de Kruskal Wallis, complementada pelo teste de comparações múltiplas quando necessário. Em todos os testes fixou-se em $5 \%$ (alfa $\leqslant 0,05$ ) o nível de rejeição da hipótese de nulidade, assinalando-se com um ou dois asteriscos $(*)(* *)$ os valores significantes.

\section{Resultados}

O peso úmido e seco do VE mostraram incremento substancial no grupo ISO em relação aos demais. Comparativamente aos grupos CON e ENA, o grupo ENA+ISO também apresentou aumento de peso, mas significativamente inferior ao encontrado no grupo ISO $(p \leqslant 0,05)$ (tab. I).

Em relação ao comportamento da PA não houve diferença entre os grupos. Já a FC apresentou um aumento significante no grupo ISO e ISO+ENA, comparativamente, ao grupo CON e ENA. O demais resultados referentes às variáveis peso do animal no final do experimento, relação peso do VE/peso do rato, relação peso úmido/peso seco do VE e proporção de pontos sobre núcleo e citoplasma estão discriminados como média e desvio padrão na tabela I.

O miocárdio no grupo ISO apresentou as fibras musculares mais volumosas (fig.1). Nas áreas próximas do endocárdio, observou-se nítida proliferação de células do tecido conjuntivo, predominantemente fibroblastos, e maior concentração de fibras colágenas entre as fibras musculares. Pela coloração do picro-sírius constatou-se que essas áreas eram ricas em colágeno do tipo I. Na mesma região também foram observadas ocasionalmente pequenas áreas de necrose. No grupo ISO+ENA a arquitetura do miocárdio apresentou-se semelhante ao do grupo CON. No entanto, em raros locais foi observado tênue proliferação de fibroblastos e de fibras colágenas (fig. 2). 


\begin{tabular}{|c|c|c|c|c|}
\hline & Controle & Enalaprilato & Isoproterenol & Enalaprilato + Isoproterenol \\
\hline Peso $(\mathrm{g})$ dos animais & $278 \pm 11$ & $281 \pm 17$ & $279 \pm 10$ & $281 \pm 11$ \\
\hline Pressão arterial - mmHg & $113,0 \pm 5,9$ & $115,5 \pm 7,2$ & $113,0 \pm 5,9$ & $115,5 \pm 8,0$ \\
\hline Frequiência cardíaca - bpm & $329 \pm 9,1$ & $323,0 \pm 12,9$ & $340,5 \pm 7,1 *$ & $338,7 \pm 13,0 *$ \\
\hline Peso seco - VE (gm) & $0,145 \pm 0,010$ & $0,139 \pm 0,010$ & $0,204 \pm 0,010^{*}$ & $0,177 \pm 0,010^{* *}$ \\
\hline Peso úmido - VE (gm) & $0,590 \pm 0,030$ & $0,590 \pm 0,050$ & $0,821 \pm 0,040^{*}$ & $0,737 \pm 0,070^{* *}$ \\
\hline Relação peso VE/peso animal & $0,521 \pm 0,060$ & $0,493 \pm 0,030$ & $0,730 \pm 0,060^{*}$ & $0,629 \pm 0,040 * *$ \\
\hline Relação peso seco/peso úmido & $24,4 \pm 1,65$ & $23,2 \pm 0,99$ & $24,5 \pm 1,28$ & $24 \pm 1,46$ \\
\hline Proporção pontos sobre núcleos e citoplasma & $85,8 \pm 1,64$ & $86,3 \pm 1,68$ & $98 \pm 1,68 *$ & $87 \pm 1,38$ \\
\hline
\end{tabular}

Nas eletromicrografias do grupo ISO foram observadas linhas $\mathrm{Z}$ aumentadas em comprimento, caracterizando aumento de sarcômeros por maior concentração de miofilamentos - espessos e delgados - bem como do espaço interfibrilar, e acúmulo de fibrilas colágenas (fig. 3). Já em relação ao grupo ISO+ENA, as eletromicrografias não apresentaram alterações ultra-estuturais: as miofibrilas estavam bem organizadas e com mitocôndrias íntegras similares ao grupo CON(fig.4).

\section{Discussão}

Satisfazendo a proposta inicial de promover HVE sem indução da hipertensão arterial, a PA sistêmica em todos os grupos estudados não variou em relação ao grupo CON. O grupo ISO apresentou aumento de cerca de 47 e $39 \%$, respectivamente, no peso seco e úmido em relação ao grupo CON. O percentual de perda líquida na comparação entre o peso úmido e seco do VE foi constante nos quatro grupos, sugerindo que a hipertrofia desencadeada pelo isoproterenol não ocorreu por edema intersticial, mas por aumento da massa miocárdica. Dado também confirmado pela microscopia ótica.

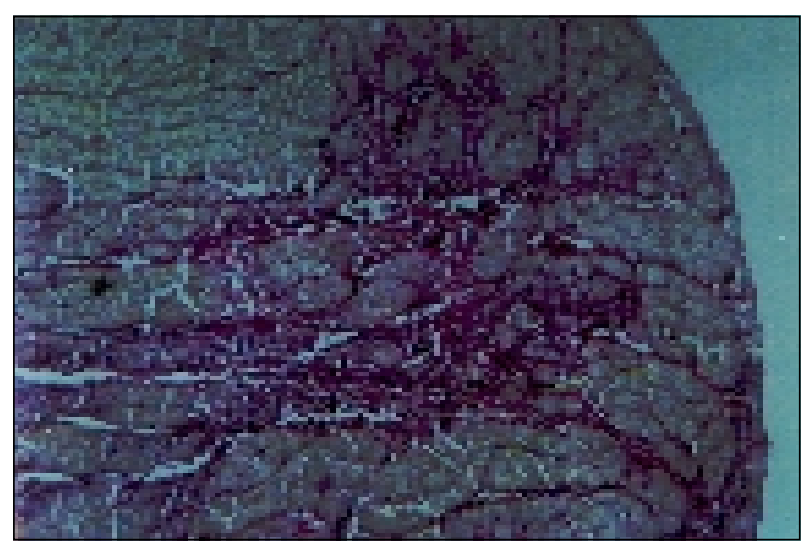

Fig. 1 - Fotomicrografia mostrando porção do coração de rato pertencente ao grupo tratado com isoproterenol (ISO). Notar cardiomiócitos hipertrofiados, cortados transversalmente. Observar ainda entre os cardiomiócitos proliferação de células do tecido conjuntivo. Hematoxilina-picro-sírius, 350x.
O isoproterenol causa hipertrofia miocárdica por ação direta nos receptores beta e pelo estímulo na produção de renina e angiotensina II, desencadeando processo hipertrófico miocárdico, através de estimulação dos receptores AT1, modulados pela proteína G que, via fosfolipase C, ativa as proteínas quinase $\mathrm{C}^{10,16}$. Outro mecanismo possível de hipertrofia do isoproterenol seria secundário à ação inotrópica e cronotrópica positiva sobre o coração. É reconhecido que o estiramento das fibras cardíacas desencadeia o processo hipertrófico via adenilciclase e AMPc, estimulando a expressão de protooncogenes ${ }^{17}$.

Quando associamos o enalaprilato ao isoproterenol a hipertrofia, tomando como parâmetro o peso do VE, foi de apenas $22,1 \%$. Isto pode ter ocorrido devido a bloqueio na formação da angiotensina II. De acordo com Nagano e $\mathrm{col}^{16}$, que dosaram a angiotensina II plasmática e tecidual no miocárdio de ratos, após o uso combinado de isoproterenol e enalaprilato, houve inibição da ECA tanto no nível sérico como no tecidual, bloqueando a formação da angiotensina II e a hipertrofia miocárdica. Também referem que a angiotensina II apresentou ação exacerbada pelo isoproterenol, elevando-se no plasma e no tecido cardíaco. Tiveram, no entanto, seus níveis diminuídos pelo enalaprilato. Estudo de

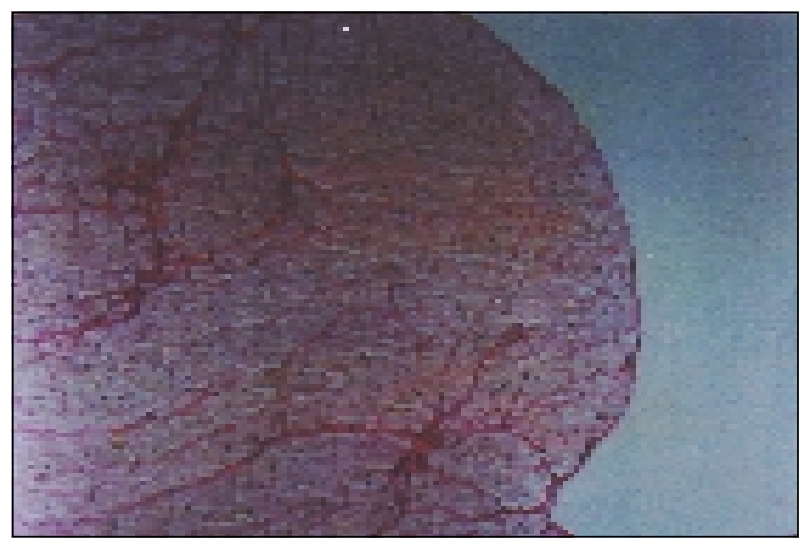

Fig. 2 - Fotomicrografia mostrando parte do coração de rato tratado com isoproterenol e enalaprilato (ISO+ENA). Notar miocárdio contendo cardiomiócitos cortados transversalmente e o endocárdio na porção mais interna. Hematoxilinapicro-sírius, 350x. 


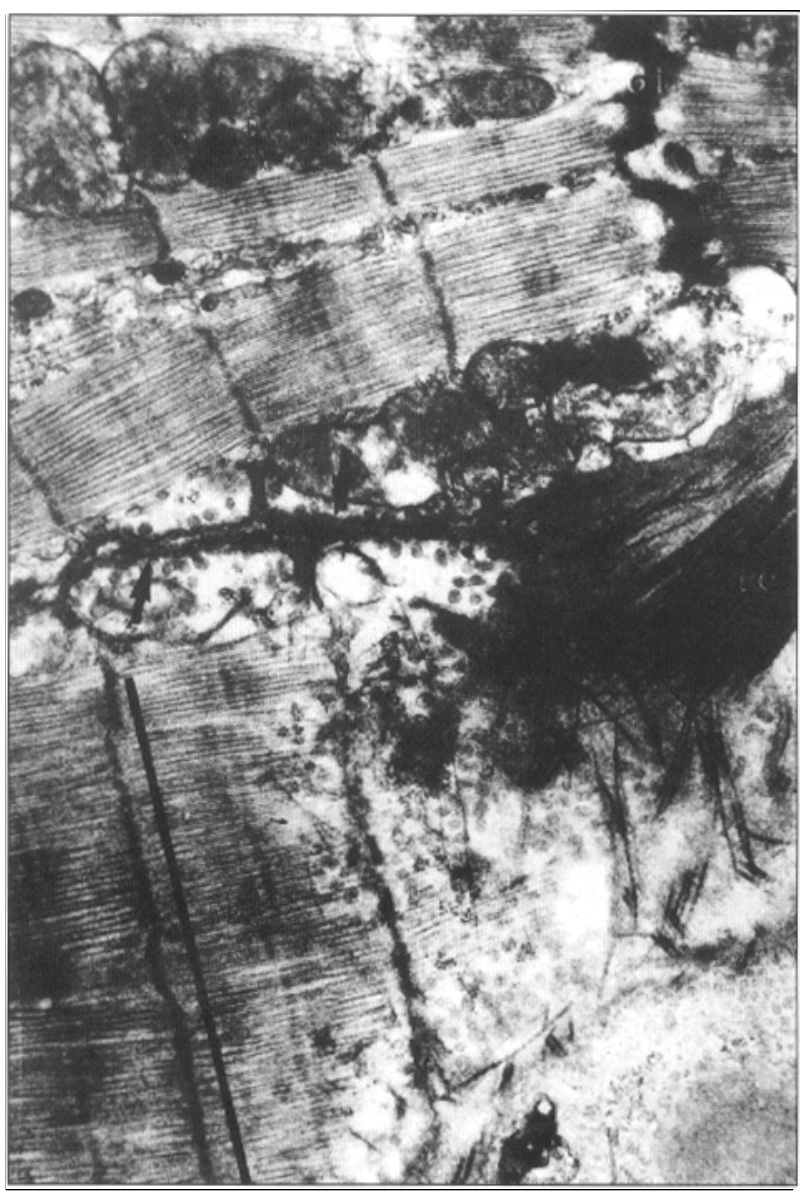

Fig. 3 - Eletromicrografia de parte de dois cardiomiócitos do grupo tratado com isoproterenol, separados por sarcolema (setas), disco intercalar característico (di), aumento do espaço interfibrilar com acúmulo de fibrilas colágenas (fc). Notar miofibrilas hipertrofiadas. Aumento: 25.380x.

Kohlmann e col ${ }^{18}$ corrobora esses resultados. Utilizando enalapril os autores diminuíram o turnover de adrenalina e noradrenalina tecidual e plasmática em ratos nãohipertensos.

Outros estudos sobre a prevenção da HVE induzida pelo isoproterenol, desenvolvidos inclusive em nosso gru-

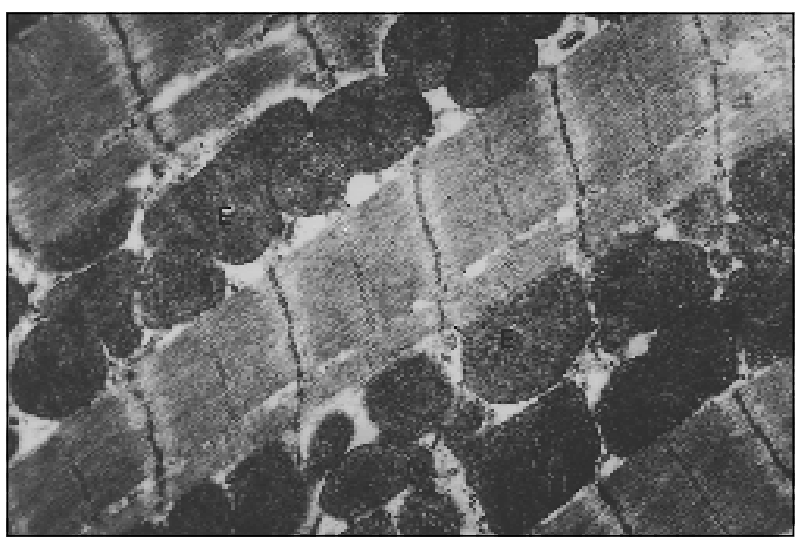

Fig. 4 - Eletromicrograia de parte de fibra muscular cardíaca do grupo tratado com enalaprilato e isoproterenol, mostrando parte de cardiomiócitos com mitocôndrias íntegras (m), miofibrilas bem organizadas. Aumento: 25.389x po, demonstraram resultados semelhantes com o verapamil, droga bloqueadora dos canais de cálcio, tornando atraente a hipótese de que a interferência na ação do isoproterenol não se faz exclusivamente no nível do receptor específico de membrana, mas, provavelmente também, com outros mediadores ${ }^{19,20}$.

Para evitar falhas técnicas relativas ao isolamento e pesagem do VE, foi realizada a avaliação morfométrica cujos resultados concordaram com as mensurações do peso ventricular. A histometria do grupo ISO apresentou aumento significante do volume dos cardiomiócitos, quando comparado aos grupos CON, ENA e ENA+ISO. Estes resultados são similares aos de Taylor e Tang ${ }^{21}$ que, empregando a mesma dose de isoproterenol utilizadas neste trabalho, observaram apenas hipertrofia dos cardiomiócitos e pouca necrose celular.

A análise histométrica neste estudo confirmou a HVE e revelou grande quantidade de colágeno, mas com escassas áreas de necrose. Por outro lado, alguns estudos com isoproterenol descreveram áreas de necrose celular extensas ou freqüentes, sugerindo que essas alterações seriam de natureza isquêmica pelo aumento abrupto e intenso do trabalho cardíaco sem o necessário aporte de oxigênio. A crítica que fazemos é que esses trabalhos utilizaram doses muito maiores de isoproterenol que a empregada no presente estudo ${ }^{22,23}$.

A formação de colágeno determina rigidez do miocárdio, levando inicialmente a uma insuficiência diastólica. Talvez seja este o primeiro mecanismo protetor da função miocárdica, evitando neste estágio a dilatação excessiva da câmara cardíaca. Posteriormente, ocorre a insuficiência sistólica, com a atrofia dos cardiomiócitos e acúmulo de fibras colágenas, isolando-os eletricamente e criando condições para o aparecimento de focos arritmogênicos 24,25 .

A hipertrofia dos cardiomiócitos e o aumento de colágeno no grupo ISO foram bem visibilizados em todas as colorações utilizadas neste estudo (hematoxilina-eosina e picro-sírius). Sob a luz polarizada nos cortes corados com picro-sírius, observamos nítido predomínio de colágeno tipo I no miocárdio do grupo ISO. Nos grupos CON e ENA, o miocárdio apresentou-se com estrutura normal. No grupo ENA+ISO, observamos além dos cardiomiócitos com estrutura praticamente normal, menor formação de colágeno tipo I, talvez pelo bloqueio da formação da angiotensina II plasmática e tecidual e ação na proteína $\mathrm{G}$ dos receptores da angiotensina II e dos canais de cálcio, o que diminuiria a ativação dos fibroblastos, células responsáveis pela produção do colágeno tipo I ${ }^{26,27}$. Assim, o miocárdio prevenir-seia dos danos causados pelo isoproterenol.

Concluindo, a administração do enalaprilato preveniu significativamente a HVE induzida pelo isoproterenol, constatada tanto através de observação com microscopia óptica, pela integridade da ultra-estrutura miocárdica, quanto através da microscopia eletrônica em relação ao aumento das mitocôndrias, tamanho dos sarcômeros e quantidade e tipo de fibras colágenas. 


\section{Referências}

1. Kannel WB, Dannenberg AL, Levy D - Population implication of left ventricular hypertrophy. Am J Cardiol 1987; 60: 851-9.

2. Brilla CG, Tan LB, Pick R - Remodeling of the rat right and left ventricle in experimental hypertension. Circ Res 1990; 67: 1355-67.

3. Lund DD, Twietmeyer AT, Schmid PG, Tomanek J - Independent changes in cardiac muscle fibers and connective tissue in rats with spontaneous hypertension, aortic constriction, hypoxia. Cardiovasc Res 1979; 13: 10-44.

4. Kannel WB, Levy D, Cupples LA - Left ventricular hypertrophy and risk of cardiac failure: insights from the Framingham study. J Cardiovsc Pharmacol 1987; 10: $135-40$.

5. Krieger EM - Neurogenic hypertension in the rat. Cir Res 1964;15: 511-21.

6. Saragoça MA, Tarazi RC - Left ventricular hypertrophy in rats with renovascular hypertension. Alterations in cardiac function and adrenergic responses. Hypertension 1981; 3(suppl II): 171-6.

7. Rossi MA, Carillo SV - Cardiac hypertrophy due to pressure and volume overload: distinctly different biological phenomenon? Int J Cardiol 1991; 31: 13342.

8. Alderman EL, Harrison DC - Myocardial hypertrophy resulting from low dosage isoproterenol administration in rats. Proc Soc Exp Biol Med 1971; 136: 268-70

9. Taylor PB, Tang Q - Development of isoproterenol induce cardiac hypertrophy. Can J Physiol 1984; 62: 384-9.

10. Bristow MR, Hershberger RE, Port JD, Monobe W, Rasmussen R - B1 and B2adrenergic receptor mediated adenylate cyclase stimulation in nonfailing and failing human ventricular myocardium. Mol Pharmacol 1989; 35: 295-303.

11. Eschenhagen T, Mende U, Nose M, Schmitz W, Scholz H, Warnholtz A, Wustel JM - Isoprenaline-induced increase in mRNA levels of inhibitory G-protein alpha-subunits in rat heart. Ach Pharmacol 1991; 343: 609-15.

12. Sympson PC - Proto-oncogenes and cardiac hypertrophy. Ann Rev Physiol 1988; 51: 189-202.

13. Reynolds ES - The use of lead citrates of high $\mathrm{pH}$ as an electron-opaque stain in electron microscopy. J Cell Biol 1963; 17: 208-13.
14. Ross MH, Romrell LJ - Histologia - Texto e Atlas. 1'ed. Bogotá: Panamericana, 1994: 283-94.

15. Junqueira LC, Bignolas G, Brentani RR - Picrosirius staining plus polarization microscopy, a specific method for collagen detection in tissue. Histochem J 1979; 11:447-55.

16. Nagano M, Higaki J, Nakamura Fet al - Role of cardiac angiotensin II in isoproterenol-induced left ventricular hypertrophy. Hypertension 1992; 19: 708-12.

17. Tarazi RC, Sen S, Saragoça M, Khairallah P-The multifactorial role of catecholamines in hypertensive cardiac hypertrophy. Eur Heart J 1982; 3(suppl A): 103 10 .

18. Kollmann Jr O, Bresnahan M, Gravas H - Central and peripheral indices of sympathetic activity after blood pressure lowering with enalapril or hydralazine in normotensive rats. Hypertension 1984; 6(suppl I): 1-6.

19. Bombig-Manzoli MT, Costa EAS, Leite DA et al - Proteção miocárdica da hipertrofia ventricular esquerda induzida pelo isoproterenol em ratos tratados com verapamil. Rev Soc Cardiol ESP 1994; 4: 102.

20. Abdel-Latif AA - Calcium mobilizing receptors, polyphosphoinositides, and the generation of second messenger. Pharmacol Rev 1986; 38: 227-72.

21. Tang Q, Taylor PB - Regression of isoproterenol-induced cardiac hypertrophy Can J Physiol Pharmacol 1984; 62: 1141-6.

22. Bloom S, Davis DL - Calcium as mediator of isoproterenol-induced myocardial necrosis. Am J Pathol 1972; 69: 459-70.

23. Benjamin Ij, Jalil JE, Tan LB, Cho K, Weber KT, Clark WA - Isoproterenol-induced myocardial fibrosis in relation to myocyte necrosis. Cir Res 1989; 65: 657-70.

24. Weber KT, Brilla CG - Pathological hypertrophy and cardiac interstitium Circulation 1991; 83: 1849-65.

25. McLenach JM, Henderson E, Morris KL, Dergie HJ - Ventricular arrhytmias in hypertensive left ventricular hypertrophy. N Engl J Med 1987; 317: 789-92

26. Weber KT, Pick R, Calil JE - Patterns of myocardial fibrosis. J Mol Cell Cardiol 1989; 21: 121-31

28. Medugorac I, Jacob R - Caracterization of left ventricular collagen in the rat Cardiovasc Res 1893; 17: 15-21. 